Instituto Internacional de Investigación y Desarrollo Tecnológico Educativo INDTEC, C.A.

DOI: https://doi.org/10.29394/scientific.issn.2542-2987.2017.0.0.8.133-153

OAI-PMH: http://www.indteca.com/ojs/index.php/Revista Scientific/oai

\title{
Fortalecimiento de la Cultura del Reciclaje y la Reutilización desde la Axiología Ambiental
}

Autora: Elizabeth Del Valle Nadales Díaz Universidad Pedagógica Experimental Libertador, UPEL elizabethnadales@hotmail.com; elizabethnadales@gmail.com

Barinas, Venezuela

\section{Resumen}

El presente trabajo de investigación, tiene como objetivo Fortalecer la cultura del reciclaje y la reutilización desde la axiología ambiental en los estudiantes y docentes de la Escuela José Francisco Jiménez, de la parroquia Ciudad Bolivia del municipio Pedraza estado Barinas, bajo el método de investigación acción participante. Por consiguiente, la misma se desarrolló en cinco fases descritas como: diagnóstico, planificación, ejecución, evaluación y sistematización. Los informantes fueron dos (2) docentes de aula, un (1) padres y representantes y dos (02) estudiantes, pertenecientes al 6to grado de la institución antes mencionada. La técnica empleada para la recolección de la información fue la observación y la entrevista, el instrumento será la guía de observación para los estudiantes y una guía de entrevista para los padres, representantes y docentes, la validez y fiabilidad de los mismos se determinó a través de la triangulación. Posteriormente, los resultados obtenidos permitieron conocer las debilidades en relación a la situación actual de la basura en la institución y la comunidad en general. A partir de las derivaciones que se generaron, se procedió a desarrollar un conjunto de acciones dirigidas a los estudiantes con el fin de fortalecer todo el contenido del cuidado del ambiente en concordancia a las nuevas políticas educativas. Al desarrollar y evaluar el estudio se llegó a la conclusión de que las acciones realizadas serán una alternativa para que los estudiantes procesen información referida a las interacciones de los sistemas ambientales, tomando acciones individuales y colectivas en la protección y defensa del ambiente para un desarrollo sostenible.

Palabras clave: cultura; reciclaje; reutilización; axiología; ambiental.

Fecha de Recepción: 23-11-2016

Fecha de Aceptación: 13-01-2017 


\title{
Strengthening Culture of Recycling and Reusing from Environmental Axiology
}

\begin{abstract}
This research aims to strengthen the culture of recycling and reuse from the environmental axiology in the students and teachers of the José Francisco Jiménez School, in the parish of Bolivia City of Pedraza state Barinas, under the action research method competitor. Consequently, it was developed in five phases described as: diagnosis, planning, execution, evaluation and systematization. The informants were two (2) classroom teachers, one (1) parents and representatives and two (02) students, belonging to the 6th grade of the aforementioned institution. The technique used for the collection of information was observation and interview, the instrument will be the observation guide for students and an interview guide for parents, representatives and teachers, the validity and reliability of the same was determined through of triangulation. Subsequently, the obtained results allowed to know the weaknesses in relation to the current situation of the garbage in the institution and the community in general. Based on the derivations that were generated, a set of actions aimed at students was developed in order to strengthen the entire content of environmental care in accordance with the new educational policies. In developing and evaluating the study, it was concluded that the actions carried out will be an alternative for students to process information related to the interactions of environmental systems, taking individual and collective actions in the protection and defense of the environment for sustainable development.
\end{abstract}

Keywords: culture; recycling; reuse; axiology; environmental.

Date Received: 23-11-2016

Date Acceptance: 13-01-2017 


\section{Introducción}

La educación ambiental se remonta a los años setenta con el fin de disminuir los problemas ambientales; cuando se denunció el impacto que se producía con el crecimiento de la población y los avances industriales, se comienza hablar de desarrollo sostenible ambiental, teniendo como preocupación la vida en el planeta para las futuras generaciones con el fin de mejorar el ambiente, llevando como bandera la acción de la educación, Venezuela no escapa de esta problemática la misma está afectando, a todos los habitantes del país, es por ello la necesidad de hacer cambios y transformaciones para evitar los factores contaminantes que han deteriorado el ambiente, revirtiendo esta situación implementando acciones ecológicas para minimizar los daños ocasionados al ambiente.

En consecuencia, es necesario hacer referencia que la educación ambiental debe vincularse con la legislación, las políticas de gobiernos, sociales y de índole económico, solo en un ambiente de crisis se consideran soluciones innovadoras por tal razón el reto es la educación en valores.

Desde esta perspectiva respetuosa con el ambiente los docentes están llamados al compromiso con la madre tierra dándole relevancia a un tema que en las escuelas y los hogares a diario puede hacerse uso el reciclaje y la reutilización siendo la misma indispensable en la acción de disminución de residuos y la reducción de basura, es evidente la necesidad de formar una cultura del reciclaje y la reutilización, el hecho de clasificar la basura según los orgánicos e inorgánicos ya es un paso, si el hombre conociera las consecuencias climáticas y de salud que generan actos indebidos en el tratamiento de residuos se estaría evitando en gran parte el problema, todo esto conduce al calentamiento global, el efecto invernadero; cruciales en la historia de la humanidad. 


\section{EI Problema}

En la actualidad se conoce al hombre como principal ente contaminante y productor de basura, es por ello, desde hace años atrás la basura se ha convertido en uno de los principales problemas ambientales de la humanidad y en su defecto, la causante de daños al ambiente, salud y sociedad. Por estas razones algunos países del mundo han creado y ejecutado proyectos que permitan la disminución de estos residuos sólidos a nivel residencial.

Es necesario señalar que, el reciclaje juega un papel importante en la conservación y protección del ecosistema, por lo tanto, es fundamental la apropiada ejecución de programas educativos sobre el reciclaje y además la puesta en práctica de acciones concretas en pro de éste, porque el correcto uso de los recursos naturales de un país depende en gran parte, de su nivel de educación ecológica. Cabe destacar, que Martínez (2012) señala que "el reciclaje se ha convertido en una alternativa socio productiva para las comunidades, que debidamente organizadas y capacitadas, pueden incidir proactivamente en mejorar sus ingresos, y calidad de vida" (pág. 12).

Por lo antes expuesto se considera, la posibilidad de aprovechar la basura y los recursos naturales de nuestro ambiente, mediante la implementación de proyectos basados en la concienciación ambiental; que permita al hombre tomar una actitud positiva en relación a dicha problemática y que mejor manera que a través del reciclaje y la reutilización, el uso racional de los recursos que nos brinda nuestra naturaleza.

En efecto, es indispensable que los individuos de una sociedad comprendan la necesidad innegable de reciclar e incluso reutilizar; cuanto tiempo tarda en degradarse una bolsa plástica un envase, entre otros, habría que esquematizar cada uno del gasto generado para una nueva adquisición y el impacto que causa al deterioro del ambiente. Al respecto, Romero (2006), señala que educación ecológica busca: 
Despertar en la población una conciencia que le permita identificarse con la problemática ambiental tanto a nivel global como local; busca identificar las relaciones de interacción e independencia que se dan entre el entorno (medio ambiente) y el hombre, así como también se preocupa por promover una relación armónica entre el medio natural y las actividades antropogénicas a través del desarrollo sostenible, todo esto con el fin de garantizar el sostenimiento y calidad de vida de las generaciones actuales y futuras (pág. 43).

De allí, que el reciclar, puede salvar los recursos naturales no renovables, ya que con los desperdicios de frutas y verduras se elaboran abonos orgánicos, una manera fácil, económica, y racional de mantener una producción de cultivos sanos y saludables sin que el ambiente sufra daño alguno y por ende al utilizar productos reutilizables, disminuye el consumo de energía y la vida de los arboles es más duradera.

En concordancia con lo anteriormente señalado el investigador considera necesario dar a conocer la importancia de reutilizar en los hogares e instituciones ya que por lo general se produce gran cantidad de residuos, Mijares (2009) señala que reutilizar "consiste en darle de nuevo utilidad a los objetos, con el mismo fin o con otros" (pág. 15). Dentro de esta perspectiva la reutilización permite que los seres humanos dependiendo del ámbito en que se desenvuelve cultive una cultura conservacionista, valorando la importancia de reutilizar, reparar, rechazar y reducir en gran medida la cantidad de basura que se produce sin contar con vertederos de basuras apropiados y el daño que causa directamente o indirectamente al ambiente.

Por lo anteriormente expuesto, todo este tipo de situaciones conducen a deducir que una de las razones del problema es la falta de conciencia ciudadana hacia la cultura del reciclaje y la reutilización para reducir en gran medida la contaminación, razones por las cuales se ha implementado la educación ambiental, en cada ámbito de la sociedad ya no solo es una exigencia a nivel educativo, es una tarea de todos. En la actualidad las 
entidades públicas y privadas se les exigen cumplir con la conservación del ambiente, e incluso en la construcción de edificios, casas locales y empresas; promueven el uso de materiales reciclados y naturales para disminuir el daño al medio ambiente, marcando tendencia en la arquitectura.

Es preciso recordar, que una de las causas primordiales que originan el problema ambiental; es la falta de conciencia ambientalista del ser humano, las políticas erradas emanadas de los gobiernos nacionales, regionales, locales e internacionales. Si nuestra cultura fuese diferente si las leyes se cumplieran y se invirtiera mayor cantidad de dinero en los problemas ambientales, fuera otra la relación del hombre con la naturaleza.

Aunado a esto, la formación ciudadana del venezolano no hace énfasis en una cultura de buena disposición y poca generación de desechos, por el contrario, se genera en promedio, por persona cerca de un kilogramo diario de desechos. Según Díaz (2002);

Venezuela requiere de alrededor de 200 rellenos sanitarios para atender la necesidad actual de disposición final de los desechos sólidos. De los que existen, sólo uno cumple con las disposiciones ambientales y sanitarias. Un poco más del $80 \%$ de la basura que se genera en Venezuela permanece expuesta en nuestras ciudades. Sus consecuencias las palpamos directa o indirectamente sobre nuestra salud, con un marcado deterioro del paisaje urbano (pág. 12).

Es por ello, la importancia del reciclaje y la reutilización de los materiales, en materia económica y ambiental, de esta manera crear conciencia, para contribuir al desarrollo sostenible de una nación por ende el futuro de las nuevas generaciones. De igual manera, la degeneración de los residuos se puede evitar a través de la reutilización de los materiales de provecho, por medio de la recuperación de los residuos, es decir, transformándolos en materia prima para el proceso productivo de otros productos. 
Tomando en cuenta lo anterior planteado es de señalar que en algunos estados como Barinas, se presentan crisis ambientales debido al modelo equivocado de desarrollo que se ha seguido, llevándolo a la existencia de situaciones críticas, donde los recursos energéticos se van agotando, de igual manera se disminuyen los espacios para la recreación, lo que genera además en la naturaleza problemas de contaminación, ruptura del equilibrio, extinción de especies, pobreza, desnutrición, consumismo, violación de los derechos humanos y otros daños alarmantes.

Con todo lo anteriormente expuesto se destaca el caso de la Escuela José Francisco Jiménez, de la parroquia Ciudad Bolivia del municipio Pedraza estado Barinas que durante las observaciones realizadas se pudo detectar la siguiente situación que se traduce en un problema para la comunidad: existe una inadecuada gestión de los residuos tanto inorgánicos como orgánicos, dentro de los primeros se encuentran el papel y plástico; y en lo segundos se encuentran los generados en el comedor (cáscaras de huevos, conchas de frutas, vegetales y verduras), producto de las actividades diarias de la cocina; además la poca sensibilización de los estudiantes al arrojar la basura en las áreas recreativas, por la ausencia de contenedores en los jardines de la institución, se acumulan gran cantidad de residuos.

Por otro lado, se pueden mencionar que la causa de esto, es la falta de presupuesto para la creación de un vertedero donde se almacenen los desechos de la institución, y por el otro la falta de programas de extensión que orienten a los docentes y estudiantes a la creación de estrategias para el manejo y aprovechamiento de los residuos los cuales han traído como consecuencia que en la comunidad no tenga cultura en cuanto al manejo y distribución de la basura, de igual manera se produce el deterioro del paisaje, así como contaminación.

Es oportuno mencionar, que desde el ámbito educativo, se pudiera crear un entorno propicio donde compartir experiencias, hablar de las 
percepciones, pensar en actuaciones positivas hacia el ambiente, no solo se trabajaría intensamente en uno de los principales problemas de la población en la cual se involucra la escuela y comunidad en la formación de una conciencia ambientalista entre sus habitantes, por qué los estudiantes de esta institución no sienten amor ni respeto por el ambiente. Dada la problemática, se hace necesario plantear la el reciclaje y la reutilización de estos residuos, como alternativa para el cuidado del ambiente. Estos planteamientos conllevan a una serie de enunciados Holopráxicos:

¿Qué estrategias utilizan los docentes para promover la cultura del reciclaje?, ¿Cómo planificar actividades para el uso del material recuperable?, ¿Cuáles cimientos epistemológicos serán necesarios para la planificación de un plan acción en cuanto a la cultura del reciclaje desde la axiología ambiental en el escenario antes citado?, ¿Qué estrategias didácticas se establecerán para sensibilizar a los estudiantes y docentes en cuanto al uso del material recuperable y el impacto que ocasiona al ambiente para los estudiantes y docentes de la escuela José Francisco Jiménez ?, ¿Cuáles aspectos se deben considerar para valorar la aplicación del plan acción hacia el fortalecimiento de la cultura del reciclaje desde la axiología ambiental en los estudiantes y docentes de la escuela José Francisco Jiménez y comunidad?

\subsection{Objetivos de la Investigación}

\subsubsection{Objetivo General}

Fortalecer la cultura del reciclaje y la reutilización desde la axiología ambiental en los estudiantes y docentes de la Escuela José Francisco Jiménez, de la parroquia Ciudad Bolivia del municipio Pedraza estado Barinas. 


\subsubsection{Objetivos Específicos}

Diagnosticar el tipo de estrategias que emplean los docentes de la Escuela José Francisco Jiménez, parroquia Ciudad Bolivia del municipio Pedraza estado Barinas en relación a la cultura del reciclaje y la reutilización.

Establecer cimientos epistemológicos para la planificación de un plan acción en cuanto a la cultura del reciclaje y la reutilización desde la axiología ambiental en el escenario antes citado.

Planificar un plan acción en cuanto a la cultura del reciclaje y la reutilización desde la axiología ambiental.

Ejecutar el plan acción en cuanto a la cultura del reciclaje y la reutilización desde la axiología ambiental en el escenario antes citado.

Sistematizar las acciones del plan acción en cuanto a la cultura del reciclaje y la reutilización desde la axiología ambiental en la Escuela José Francisco Jiménez, parroquia Ciudad Bolivia del municipio Pedraza estado Barinas.

\section{Referentes Teóricos}

\subsection{Teoría Ecológica de Bronfenbrenner}

El modelo ecológico de desarrollo humano de Bronfenbrenner (1979), ofrece una compresión de la compleja y permanente interacción de las personas con sus ambientes más o menos inmediatos. Explica que el desarrollo humano se lleva a cabo a través de "una acomodación recíproca progresiva entre un ser humano activo, en desarrollo, y las propiedades cambiantes de los escenarios inmediatos en los que está viviendo" (pág. 40). Igualmente, manifiesta que el desarrollo implica cambios perdurables que se transmiten a otros lugares en momentos diferentes, dándose estos cambios en su percepción, por cuanto la persona amplía y enriquece la forma de percibir el entorno, así como su acción, al evolucionar, cambia tanto la 
variedad como complejidad de las actividades que realiza en distintos ambientes.

Así mismo, considera a la persona en desarrollo como organismo activo en continua transición ecológica porque produce cambios en su estatus o rol social, en su entorno o en ambos a la vez. Hace crítica radical al estudio del desarrollo de los individuos al margen de los contextos en que se encuentran y como esto influye al ambiente ecológico como un conjunto de estructuras seriadas y estructuradas en diferentes niveles.

\subsection{El Reciclaje}

Hace menos de dos siglos en los países desarrollados los trapos viejos, los escombros o el estiércol fueron materiales valiosos para la fabricación de papel, la construcción o la agricultura respectivamente. Pero en el último cuarto de siglo XXI la práctica cada vez más extendida de recuperar lo utilizado responde a un límite de una parte de los recursos naturales ante la fabricación y elaboración de objetos con material recuperable que caducan, cuya finalidad es darle un nuevo uso.

A este respecto, el Reciclaje corresponde a la transformación física, química o biológica de algunos desechos, de modo que se obtenga una nueva materia prima con la que se elabora los mismos productos o diferentes. Este también es una nueva alternativa de soluciones provechosas en el manejo de residuos que la sociedad de consumo ha descartado por considerarlos inútiles.

Así también lo expresa un contexto de opinión de Ciencias naturales Ecosistema (2003), señala que "el reciclaje disminuye la cantidad de basura, prolonga la vida útil, preserva los recursos naturales, se economiza energía y minoría la contaminación" (pág. 13). En tal sentido la propuesta formula el desarrollo de estrategias didácticas que contribuyan al mantenimiento la descontaminación del ambiente y el poder despertar la creatividad cognitiva y motora fina del individuo. 


\subsection{Reutilización de Materiales}

Desde hace unos años, se ha reiniciado la toma de conciencia mediante acciones que tienen como fin concientizar que la tierra, como hogar de todos, es responsabilidad de todos sus habitantes. De esta manera al involucrar a cada individuo, debe modificarse la realidad, transformando dicha "casa" y tomando medidas certeras para preservarla, lo cual representará progreso y calidad de vida.

Surge de este modo, la reutilización de materiales, para Sánchez (2008) es el "producto resultante del Reciclaje, puede extraerse de prácticamente todas las materias que se someten al Reciclado, con la excepción de los materiales más contaminantes, como son las pilas o la basura nuclear, para los que aún no existen procesos eficiente de reutilización" (pág. 23), es importante resaltar que el fin último de reutilizar materiales, es reducir el volumen de desechos sólidos, este refiere la clasificación de dichos residuos de acuerdo a su material, ya sea vidrio, plástico, papel, cartón, metal, entre otros. En este orden de ideas, puede reutilizarse el material recolectado, aplicando determinado proceso productivo para poder ser utilizado nuevamente.

\subsection{Transformación de Residuos}

Es cualquier objeto, material, sustancia o elemento sólido, semisólido, líquido o gaseoso resultante del consumo o uso de un bien en actividades domésticas, industriales, comerciales, institucionales o de servicios, que el generador abandona, rechaza o entrega y que es susceptible de aprovechamiento o transformación en un nuevo bien, con valor económico o de disposición final.

El término generalmente de acuerdo a lo que expresa Solis (2009) "se refiere a los materiales producidos por la actividad humana, $y$, en general, para reducir sus efectos sobre la salud y el medio ambiente" (pág. 15). La gestión 
de los desechos es también llevada a cabo para recuperar los propios recursos de dichos residuos.

Los residuos sólidos se dividen en aprovechables y no aprovechables. Igualmente, se consideran como residuos sólidos, entre otros, aquellos provenientes del barrido, limpieza de áreas y vías públicas, corte de la maleza y poda de árboles. El manejo de desechos sólidos es la gestión de los residuos, la recogida, el transporte, tratamiento, reciclado y eliminación de los materiales de desecho.

\subsection{Basura}

La basura es cualquier residuo inservible o material no deseado que se tiene que desaparecer. Según Colomar y Gallardo (2012) define la basura como residuo a "aquellas materias generadas en las actividades de producción y consumo que no han alcanzado un valor económico en el contexto en el que son producidas" (pág. 6). Constantemente, se están generando muchos productos para satisfacer las necesidades de una sociedad cada vez más materializada. Al aumentar la población y la reacción de nuevos objetos, se incrementa también el desecho de esos productos cuando ya no son utilizados. Al dejar de ser utilizados y además mezclados con otros, los productos se convierten en basura. La Basura no existe por naturaleza, sino que es generada por el ser humano debido a la irresponsabilidad, malos hábitos o falta de cultura. Se genera diariamente, en todos los entornos: en la escuela, la oficina, la fábrica, la casa, entre otros.

En este sentido, Godoy (2010) hace mención a que "el manejo de la basura es el término empleado para designar al control humano de recolección, tratamiento y eliminación de los diferentes tipos de residuos (pág. 12). Es decir, estas acciones se realizan para reducir el nivel de impacto negativo de la basura sobre el medio ambiente y la sociedad. De igual manera, a veces no se realiza un esfuerzo mayor para no generar basura o bien, para 
evitar que se mezcle y acumule. Sin embargo, en las instituciones educativas deberían existir grupos de personas que se encarguen de recolectar la basura, concentrarla en un solo lugar y hacerla llegar a algún centro de acopio para contribuir a fomentar la cultura del reciclaje.

\subsection{Axiología Ambiental}

La complejidad de la problemática ambiental necesita respuestas y soluciones, por ello exige que el hombre reaccione sobre el conocimiento de la realidad ambiental, identificación de sus problemas y la sensibilización ambiental. En ese sentido el Ministerio del Poder Popular para la Educación (2007), sustenta, incorpora e incluye la educación ambiental como eje integrador del conocimiento; medio a través del cual se promueve en el individuo la toma de conciencia en cuanto al tema ambiental se refiere, plantea la necesidad de fomentar una conciencia ciudadana mediante el desarrollo de una cultura de valores ambientalistas, en la que la escuela y el docente están llamados a lograr este objetivo.

Por tanto, resulta indispensable motivar a la población, hacia el cuidado del ambiente, promoviendo la participación de todos en la solución de los problemas ambientales que se presentan, con una administración eficiente y racional de los recursos naturales, Pabón (2003) señaló:

Ante el reconocimiento de la sociedad global de los desequilibrios causados sobre el entorno natural, surgió la perspectiva educativa ambiental, para el logro del desarrollo humano sostenible, que busca emprender la estrategia de la educación ambiental en todos los niveles, conduciendo a la institucionalización de la formación ambiental. Paralelamente desde el campo educativo se inició una construcción conceptual y metodológica que ha permitido realizar programas de educación ambiental, en este contexto se suscita un análisis sobre esta dimensión de la formación integral (pág. 72). 
Para la consecución de esta tarea es necesario propiciar escenarios para el diálogo, la discusión y la reflexión colectiva, con miras a generar una construcción social del conocimiento del saber ambiental, definición de responsabilidades en la promoción del espíritu de colaboración, de complementariedad por un desarrollo integral de la persona, donde se toma en cuenta la realidad socio-ambiental como insumo para la construcción colectiva de procesos de aprendizaje.

\section{Marco Metodológico}

La metodología es el camino estratégico a seguir para iniciar, ejecutar y finalizar el proceso investigativo con éxito, en ella se incluye el enfoque, tipo de investigación, diseño, las técnicas y procedimientos que serán utilizados para llevar a cabo la indagación, el marco metodológico constituye la esencia de la investigación, en él se plantea el desarrollo del proceso investigativo.

\subsection{Naturaleza de la Investigación}

La metodología implementada en cuanto al enfoque fue cualitativa, la cual, según Martínez (2009):

No se trata del estudio de cualidades separadas o separables; se trata, pues, del estudio de un todo integrado que forma o contribuye primordialmente una unidad de análisis y que hace que algo sea lo que es: una persona, una entidad étnica, social, empresarial, un producto determinado, etc.; aunque también cabe la posibilidad de estudiarse una cualidad específica, siempre que se tenga en cuenta los nexos y las relaciones que tienen con el todo, los cuales contribuyan a darle su significación propia (pág. 66).

En virtud de lo anteriormente citado, la investigación cualitativa permite el estudio de la naturaleza de las realidades, integrado en función a conocer el contexto social. Es decir, llega a describir fenómenos, logrando en algunos casos ser partícipes de la transformación. 


\subsection{Tipo y Diseño de Investigación}

En consideración, a las características propias del hecho observado y dar respuesta a la necesidad de fortalecer la cultura del reciclaje y la reutilización desde la axiología ambiental en los estudiantes y docentes de la Escuela José Francisco Jiménez, de la parroquia Ciudad Bolivia del municipio Pedraza estado Barinas.

El estudio se ejecutó a través de la investigación acción participante, esto significa que el investigador participa también en el estudio y se encuentra dentro del contexto observado el fenómeno, además comparte con los estudiantes. Al respecto Hurtado y Toro (2005) define la investigación acción participante, como:

Una forma de investigación social, y se caracteriza por el hecho de que la acción, más que un producto de intereses particular del investigador, surge como necesidad de un grupo y es llevada a cabo por los involucrados en la situación a modificar, escenario que permite al investigador actuar como facilitador del proceso (pág. 122).

De acuerdo con lo expresado anteriormente se hace pertinente la presente investigación, puesto que el propósito consiste en la participación de la investigadora en el logro del fortalecer la cultura del reciclaje y la reutilización desde la axiología ambiental en los estudiantes y docentes de la Escuela José Francisco Jiménez, de la parroquia Ciudad Bolivia del municipio Pedraza estado Barinas.

\subsection{Fases de la Metodología}

En la investigación acción se plantea la necesidad generar conocimientos que puedan ser útiles para resolver problemas del ámbito educativo y faciliten a sus miembros la formación para enfrentar y resolver otras debilidades que se presenten. A partir de estos propósitos el presente estudio se desarrollará tomando en consideración las fases diseñadas por 
Hurtado y Toro (2005) este proceso se desarrolla atendiendo a las siguientes fases: (1) Diagnóstico. (2) Planificación (3) Ejecución, (4) Evaluación y (5) Sistematización.

\subsection{Informantes}

De acuerdo a lo expuesto, la elección de los informantes, en el enfoque cualitativo con el tipo de investigación acción participante, pretende que el investigador seleccione con exactitud, los informantes que darán respuestas a las acciones del problema en estudio, y son participantes esenciales del proceso. A tal efecto los informantes para la investigación lo constituyeron dos (2) docentes de aula, un (1) padres y/o representantes y dos (02) estudiantes, pertenecientes al 6to grado de la Escuela Básica José Francisco Jiménez, ubicada en el Municipio Pedraza del Estado Barinas, durante el año escolar 2015-2016.

\subsection{Técnicas de Recogida de Información}

Las técnicas de recolección de datos en la investigación cualitativa son diversas; porque se trata de obtener el máximo de información pertinente al estudio, que genere interpretación más aproximada de la realidad y de los factores incidentes en ella. Por tanto, en este caso se utilizará como técnica la entrevista y la observación directa.

De igual forma la observación para Anastas, (2005) es "la observación es formativa y constituye el único medio que se utiliza siempre en todo estudio cualitativo. Podemos decidir hacer entrevistas o sesiones de enfoque, pero no podemos prescindir de la observación" (pág. 16).

\subsection{Validez de la Información}

En la investigación cualitativa, la validez es la de mayor peso en la investigación, porque permite recoger las informaciones y captar cada 
escenario desde el punto de vista de estudiar, analizar e interpretar la realidad proporcionando la fiabilidad de las conclusiones. Asimismo, García (2004) señala que "la validez concierne a la exactitud con que las conclusiones representan efectivamente la realidad" (pág. 139). En este sentido la validez hace referencia a la claridad, consistencia interna y externa del instrumento, en la cual los datos se comparar y analizan constantemente para asegurar la articulación entre dimensiones y realidad.

\subsection{Confiablidad}

La fiabilidad de esta investigación queda revelada en la medida en que los hallazgos puedan destinarse a otros individuos, de esta forma resulta probada la transferibilidad de las referencias que fueron proporcionada en el diagnóstico, esta expresión la afirma García (2004), al conceptualizar la fiabilidad como: "...un proceso empírico para verificar el grado de similitud entre el contexto del evaluador y lo evaluado" (pág. 140). De lo antes expuesto, es importante resaltar que la pensadora detalló elementos muy sustanciales y explicativos de todos los fenómenos que se suscitaron en el entorno escolar y la actuación del docente en el modelo ecológico que demuestra al direccionar la práctica educativa.

\subsection{Técnicas de análisis de la información}

Las técnicas y análisis de la información, permiten procesar la información obtenida en las guías temáticas de la entrevista en profundidad, pero la misma debe ser organizada y esquematizada, trascribiendo todos los contenidos, entendiéndose según Martínez (2009), "como los relatos escritos, extraídos de las grabaciones y realidades de las situaciones concretas" (pág. 23). Por estas razones, se debe organizar la información de manera escrita citando las fuentes de las mismas. 


\section{Conclusiones}

Luego de aplicadas las técnicas e instrumento para diagnosticar la necesidad de reciclar y reutilizar materiales de provecho para el fortalecimiento del cuidado del ambiente se pudo constatar al contrastar tanto la entrevista de los docente como la de los padres y representantes que no se están llevando a cabo acciones dirigidas a la conservación del ambiente en este sentido, se observa como existe divergencia en algunas apreciaciones con respecto a la participación por cuanto los docentes alegan que es poca la participación de los padres y ellos a su vez manifiestan que no han sido llamados a participar.

Desde el punto de vista de la participación de los padres, representantes y responsables en las actividades que se realicen en las instituciones educativas, señala Hanson (2013), "no cabe duda que la Ley no solamente lo facilita, sino que lo exige; así, que toda planificación que se desarrolle para cumplir este cometido, no solamente es necesaria sino apegada al espíritu de la Ley y, por lo tanto, absolutamente viable" (pág. 2).

En este sentido toda actividad que sea planificada en la escuela a nivel general o por los docentes, debe contar con la participación de los padres y representantes de modo que ellos puedan compartir con sus hijos el proceso de aprendizaje que sea cual fuere la actividad constituye un aporte significativo.

Desde esta perspectiva es indispensable educar en valores ambientales teniendo en consideración la temprana edad, enseñando hábitos positivos para la preservación del ambiente, siendo los niños el futuro de un país.

\section{Referencias}

Anastas, P. (2005). La Protección del Medio Ambiente y Los Recursos Naturales en la Nueva Constitución del Perú. Revista del Instituto de 
Investigación de la Facultad de Ingeniería Geológica, Minera, Metalúrgica y Geográfica.

Bronfenbrenner, U. (1979). Ecología él de Desarrollo Humano: Los experimentos de la naturaleza y Diseño Cambridge, MA: Harvard University Press.

Colomar y Gallardo (2012). Naturaleza y función de las actitudes ambientales. Estudios de Psicología. España. Editorial. Pirámide.

Cuevas, (2009). Influencia de los hábitos alimentarios en el estado nutricional del adulto mayor. Pontificia Universidad Javeriana Facultad de Ciencias Carrera de Nutrición y Dietética. Colombia.

Díaz, F. (2002). Estrategias Docentes para un Aprendizaje Significativo. Caracas: OBL Editores.

García, M. (2004). Hacia el Perfeccionamiento de la escuela primaria. La Habana: Editorial Pueblo y Educación.

Godoy, M. (2010). Desarrollo Sostenible. 1era Edición. Editorial UPC. Barcelona, España.

Hanson, M. (2013). La Construcción Sustentable de una Nueva Era. Diario VEA. Caracas, Venezuela.

Hurtado, I. Toro, J. (2005). Paradigma y métodos de investigación en tiempos de cambio. Los libros de El Nacional. Colección Minerva.

Martínez J. (2012). El reciclaje. La forma más fácil de mantener nuestro planeta vivo. Gestión Ambiental. Universidad de Palermo.

Martínez, M. (2009). Epistemología de la investigación cualitativa. México: McGraw-Hill.

Mijares, C. (2009). Desarrollo Endógeno y Educación. Estrategia de Transformación. Editorial Panapo de Venezuela.

Ministerio del Poder Popular Para La Educación (2007). Currículo Básico Nacional Bolivariano. Subsistema de Educación Primaria. Caracas Venezuela. 
Pabón, M. (2003). Contexto Internacional de la Educación Ambiental. Revista De Ciencias Humanas. Bogotá, Colombia: Universidad Tecnológica de Pereira.

Romero, L. (2006). Despertar en la Población una Conciencia del Valor Ambiente. El papel de los valores desde el modelo Hall-Tonna. En Educadores, Año 39, Volumen 40-41, № 182 -183. España.

Sánchez, L. (2008). La Orientación Participativa: Una experiencia en la Subdirección de Investigación y Postgrado de la UPEL-IPB. Solis, L. (2009). El Aprendizaje Cooperativo como Estrategia de Enseñanza y Aprendizaje. España: Editores Siglo XXI. 


\section{Elizabeth Del Valle Nadales Díaz}

e-mail: elizabethnadales@hotmail.com; elizabethnadales@gmail.com

Técnico Superior en Educación Integral (Instituto

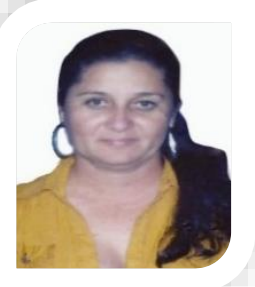

Universitario de Tecnología Coronel Agustín Codazzi,

Barinas). Licenciada en Educación Integral

(Universidad Católica Cecilio Acosta, Maracaibo).

Magíster en Educación Ambiente y Desarrollo

Universidad Pedagógica Experimental Libertador,

Barinas (FALTA). Docente titular (Escuela Básica Nacional José Francisco Jiménez).

El contenido de este manuscrito se difunde bajo una Licencia de Creative Commons ReconocimientoNoComercial-Compartirlgual 4.0 Internacional 